\title{
Electrochemical behaviour of chloroplast, thylakoid, and PSII on the chitosan modified glassy carbon electrode
}

\author{
Jinglun Zhao ${ }^{1, *}$, Suqing Zhu ${ }^{2, *}$, Lingjuan Tang ${ }^{3}$, Hao Huang ${ }^{1}$, Mengru Ming ${ }^{2}$, Wenping Huang ${ }^{2}$, \\ Haiying $G u^{1, *}$, Lijun Sun ${ }^{2, *}$ \\ ${ }^{1}$ School of Public Health, Nantong University, Nantong, Jiangsu, 226019, China. \\ ${ }^{2}$ School of Life Sciences, Nantong University, Nantong, Jiangsu, 226019, China. \\ ${ }^{3}$ Analysis and Testing Center, Nantong University, Nantong 226019, Jiangsu, China

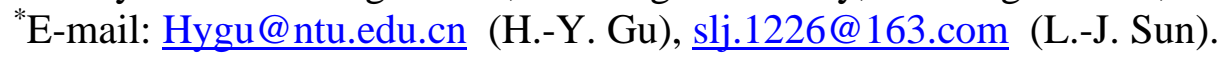

doi: $10.20964 / 2020.10 .52$

Received: 8 June 2020 / Accepted: 30 July 2020 / Published: 31 August 2020

\begin{abstract}
One primary and critical issue in a photosynthesis process is the absorption, transfer, and conversion of light energy in chloroplast. Although previous studies have shown that the absorption, transfer and conversion of light energy are involved in a series of ordered pigment protein complexes, there is still lack of direct evidence on the electron transfer mechanism within a chloroplast. In this study, chloroplast, thylakoid, and photosystem II (PSII) were assembled on the chitosan modified glassy carbon electrodes to explore the corresponding photosynthetic properties. The scanning electron microscopic and UVvisible spectral analysis showed that the chloroplast, thylakoid, and PSII were successfully modified on the electrode surfaces with their photosynthetic activity. The electrochemical features of chloroplast, thylakoid, and PSII were investigated through cyclic voltammetry. The results showed that only chloroplast modified electrodes didn't present any electrochemical activity, while the chloroplast modified electrodes soaked in water (hypotonic treatment) for 1 to 3 hours presented a couple of quasireversible redox peaks. Both thylakoid and PSII modified electrodes without hypotonic treatment showed similar redox peaks as that of chloroplast modified one, suggesting that the electrochemical signal sources for chloroplast, and thylakoid are out of PSII. In summary, this study not only explained the direct electrochemical signal source of the chloroplast, but also provided a new strategy to study the electron transfer process inside an organelle or cell.
\end{abstract}

Keywords: Direct electrochemistry; Modified electrode; Chloroplast; Thylakoid; Photosystem II

\section{$\underline{\text { FULL TEXT }}$}

(C) 2020 The Authors. Published by ESG (www.electrochemsci.org). This article is an open access article distributed under the terms and conditions of the Creative Commons Attribution license (http://creativecommons.org/licenses/by/4.0/). 